\title{
Direct Interaction of $\alpha$-Synuclein and AKT Regulates IGF-1 Signaling: Implication of Parkinson Disease
}

\author{
Ji-Yun Chung a, d Su-Jin Lee ${ }^{a, d}$ Sun-Hye Lee ${ }^{a}$ Youn Sang Jung ${ }^{a} \quad$ Nam-Chul Hab \\ Wongi Seolc Bum-Joon Park ${ }^{\mathrm{a}}$ \\ a Department of Molecular Biology, College of Natural Science, ${ }^{b}$ College of Pharmacy, Pusan National University, and \\ 'The Institute of Brain Science and Technology, Inje University, Busan, Republic of Korea
}

\section{Key Words}

$\alpha$-Synuclein $\cdot$ AKT $\cdot$ Parkinson's disease $\cdot$ IGF-1 signaling

\begin{abstract}
Genetic mutation of $\alpha$-synuclein ( $\alpha$-SYN) is clearly verified as the causal factor of human and mouse Parkinson's disease. However, biological function of $\alpha$-SYN has not been clearly demonstrated until now. In this investigation, we reveal that $\alpha$-SYN is a co-regulator of growth factor-induced AKT activation. Elimination of SYN reduces the IGF-1-mediated AKT activation. Similarly, mutant SYN suppresses the IGF-1-induced AKT activation. Wild-type SYN can interact with AKT and enhance the solubility and plasma localization of AKT in response to IGF-1, whereas mutant $\alpha$-SYNs do not interact with AKT. In addition, elevated expression of SYN blocks the AKT activation. We also find that si-RNA against $\alpha$-SYN abolished the protective effect of IGF-1 against DNA damage-induced apoptosis. Our result strongly indicates that Parkinson's disease, induced by $\alpha$-SYN mutation, is evoked by deregulation of the AKT-signaling cascade. C Copyright $\odot 2011$ S. Karger AG, Basel
\end{abstract}

Ji-Yun Chung and Su-Jin Lee equally contributed to this work.
Parkinson's disease (PD) is the second leading neurodegenerative disease [1]. As well confirmed, dopaminergic motor neurons in substantia nigra are reduced through cell death $[1,2]$. Thus, PD patients suffer from movement disorder, resting tremor, rigidity, bradykinesia, and postural instability [1]. Despite an exponential increase of PD incidence, the pathological cause of this disease has not been clearly demonstrated. In several cases, genetic mutations have been suggested to be related with PD [3, 4]. Until now, 15 kinds of responsible chromosomal loci have been suggested [3, 4]. Among them, two sites including amplification of chromosome 4 are revealed as $\alpha$-synuclein $(\alpha$-SYN) $[1,5,6]$. Although it is clear that overexpression or genetic mutation of $\alpha$-SYN can evoke the PD $[6,7]$, until now the physiological or pathological role of this protein has not been clearly demonstrated. In this respect, it has been proposed that aggregation of $\alpha$-SYN (Lewy body) [8] is a frequently detected pathological feature of PD. In fact, several kinds of proteins including synphilin have been identified as coaggregating proteins with $\alpha$-SYN in Lewy body $[9,10]$. However, the pathological or physiological relevance of these proteins with PD has not been demonstrated. In addition, it has been suggested that $\alpha$-SYN-deficient mice also show the defect in the nigrostriatal dopamine system

\section{KARGER \\ Fax +4161306 1234 \\ E-Mail karger@karger.ch}

www.karger.com
(C) 2011 S. Karger AG, Basel

1424-862X/11/0192-0086\$38.00/0

Accessible online at:

www.karger.com/nsg
Bum-Joon Park, MD

Department of Molecular Biology, College of Natural Science

Pusan National University

Busan (Republic of Korea)

Tel. +82 51510 2220, E-Mail bjpark1219@ pusan.ac.kr 
[2]. This fact indicates that loss of $\alpha-S Y N$ can lead to dysfunction of the dopaminergic nerve system, which is known to occur by mutation or amplification of $\alpha-S Y N$.

On the other hand, it has been suggested that growth factors including IGF-1 can ameliorate the pathological symptoms of PD $[11,12]$. Also, other PD-related genes including DJ-1 or Omi/HtrA2 are linked with apoptosis in a direct or indirect manner [13-16]. These previous results strongly suggest that the biological function of $\alpha$ SYN would be related with apoptosis regulation.

To address the relevance between $\alpha$-SYN and IGF-1 signaling and demonstrate the physiological role of $\alpha$ SYN, we checked the effect of wild-type (WT) and mutant $\alpha$-SYN in IGF- 1 signaling and found that $\alpha$-SYN is required for proper activation of AKT and IGF-1.

\section{Materials and Methods}

\section{Cell Lines and Reagents}

Human cell lines were obtained from American Type Culture Collection (Manassas, Va., USA) maintained in RPMI or DMEM liquid media (containing $10 \% \mathrm{FBS}$ and $1 \%$ antibiotics) at $37^{\circ} \mathrm{C}$ in humidity chambers with $5 \% \mathrm{CO}_{2}$ supplement. Normal human fibroblast (GM 00038, 9 years old) was obtained from Coriell Cell Respiratory Collection (Camden, N.J., USA). IGF-1 was supplied by R\&D Systems, Inc. (Minneapolis, Minn., USA). Adriamycin was obtained from Sigma-Aldrich (St. Louis, Mo., USA). Antibodies against $\alpha$-SYN, actin, GST, lamin A/C, and HA were provided by Santa Cruz Biotechnology, Inc. (Santa Cruz, Calif., USA). Antibodies against p-AKT (Thr308 and Ser473) and total AKT (t-AKT) were obtained from Cell Signaling Technology, Inc. (Danvers, Mass., USA). Secondary antibodies were purchased from Pierce (Thermo Fisher Scientific, Inc., Rockford, Ill., USA). Other general reagents were obtained from Sigma-Aldrich.

\section{Vectors and si-SYN}

SYN expression vectors were obtained for Dr. Seol (Inje Univ. Korea) and AKT and AKT-KD expression vectors were provided by Dr. Chung (Pusan Natl. Univ. Korea). According to a previous report [17], we generated two kinds of si-SYN. For transfection, Jet-pei reagent was used. Transfection was performed following the manufacturer's protocol. In brief, cells were seeded and incubated with DNA and reagent mixture for $3 \mathrm{~h}$ in a serum-free condition. After washing with serum-free media, cells were incubated with complete media for an additional $21 \mathrm{~h}$. Then, after washing, cells were incubated with IGF-1, NGF-1 or adriamycin.

\section{Protein Analysis and Immunoprecipitation}

Protein expression was determined by general Western blot (WB) analysis [18]. In brief, cells were harvested using RIPA (containing protease inhibitor cocktail). After centrifugation (12,000 rpm for $10 \mathrm{~min}$ ), supernatant was collected and subjected into SDS-PAGE after denaturation with an SDS sample buffer. Precipitated material was dissolved with benzonase containing RIPA and subjected into SDS-PAGE. For immunoprecipitation (IP),
AKT-transfected cell lysates and SYN-transfected cell lysate were mixed and incubated with anti-SYN antibody for $2 \mathrm{~h}$ at room temperature (RT). Sequentially, protein A/G-agarose bead was added and incubated for $1 \mathrm{~h}$. After washing twice with PBS, precipitated material was analyzed by SDS-PAGE.

\section{GST Pull-Down}

A detailed protocol of GST-fused protein generation has been described previously [18]. In brief, the $\mathrm{PH}$ domain and kinase domain of AKT were cloned into pET vector through PCR and expressed in Escherichia coli. For binding assay, GST bead-conjugated AKT or SYN was incubated with cell lysates for $1-2 \mathrm{~h}$ at RT. After centrifugation, collected proteins were subjected into SDSPAGE and WB analysis.

\section{MTT Assay}

To address the effect of SYN or si-SYN on cell viability, cells were transfected with wild, mutant SYN or si-SYN for $24 \mathrm{~h}$. For MTT assay, cells were incubated with $0.5 \mathrm{mg} / \mathrm{ml}$ of MTT solution for $4 \mathrm{~h}$ at $37^{\circ} \mathrm{C}$. After removing excess solution, the precipitated materials were dissolved in $200 \mu \mathrm{l}$ DMSO and quantified by measuring absorbance at $540 \mathrm{~nm}$. To examine the protective effect of IGF-1 against DNA damage, SK-N-SH cells were transfected with WT-SYN or si-SYN for $24 \mathrm{~h}$. After washing, cells were incubated with $0.2 \mu \mathrm{g} / \mathrm{ml}$ of adriamycin alone or with IGF-1 $(5 \mathrm{ng} / \mathrm{ml})$ for $6 \mathrm{~h}$ and subjected to MTT assay [18].

\section{Far-WB Analysis}

For determination of the direct interaction between AKT and SYN, we performed the far-WB analysis [18]. AKT or AKT-KD fixed membrane was incubated with WT-SYN, A30P-SYN, and A53T-SYN recombinant protein for $4 \mathrm{~h}$ at $4^{\circ} \mathrm{C}$. After washing, membranes were incubated with anti-GST antibody. After detection using ECL, membranes were reprobed with AKT antibody.

\section{Results}

\section{Mutant $\alpha$-SYN Suppressed the IGF-1-Induced AKT Activation}

To address the role of $\alpha$-SYN, we first examined the engagement of $\alpha$-SYN in IGF-1-induced AKT activation. As well confirmed, IGF-1 could induce the AKT phosphorylation at Ser473 (fig. 1a). However, mutant $\alpha$-synuclein (MT-SYN) blocked the activation of AKT in 293 cells (fig. 1a). To confirm this, we measured the AKT activation in the human neuroblastoma cell line, SK-N-SH, and obtained a similar result that MT-SYN blocked the AKT activation, whereas wild-type $\alpha$-SYN (WT-SYN) could promote the activation (fig. 1b). We could also observe the enhancement of AKT activity by monitoring the Ser9 phosphorylation of GSK3 $\beta$ (fig. 1b). Since AKT activation is achieved by cytoplasmic localization, where AKT is phosphorylated by PDK or PI3K [19], we examined the effect of SYN on AKT localization using immu- 

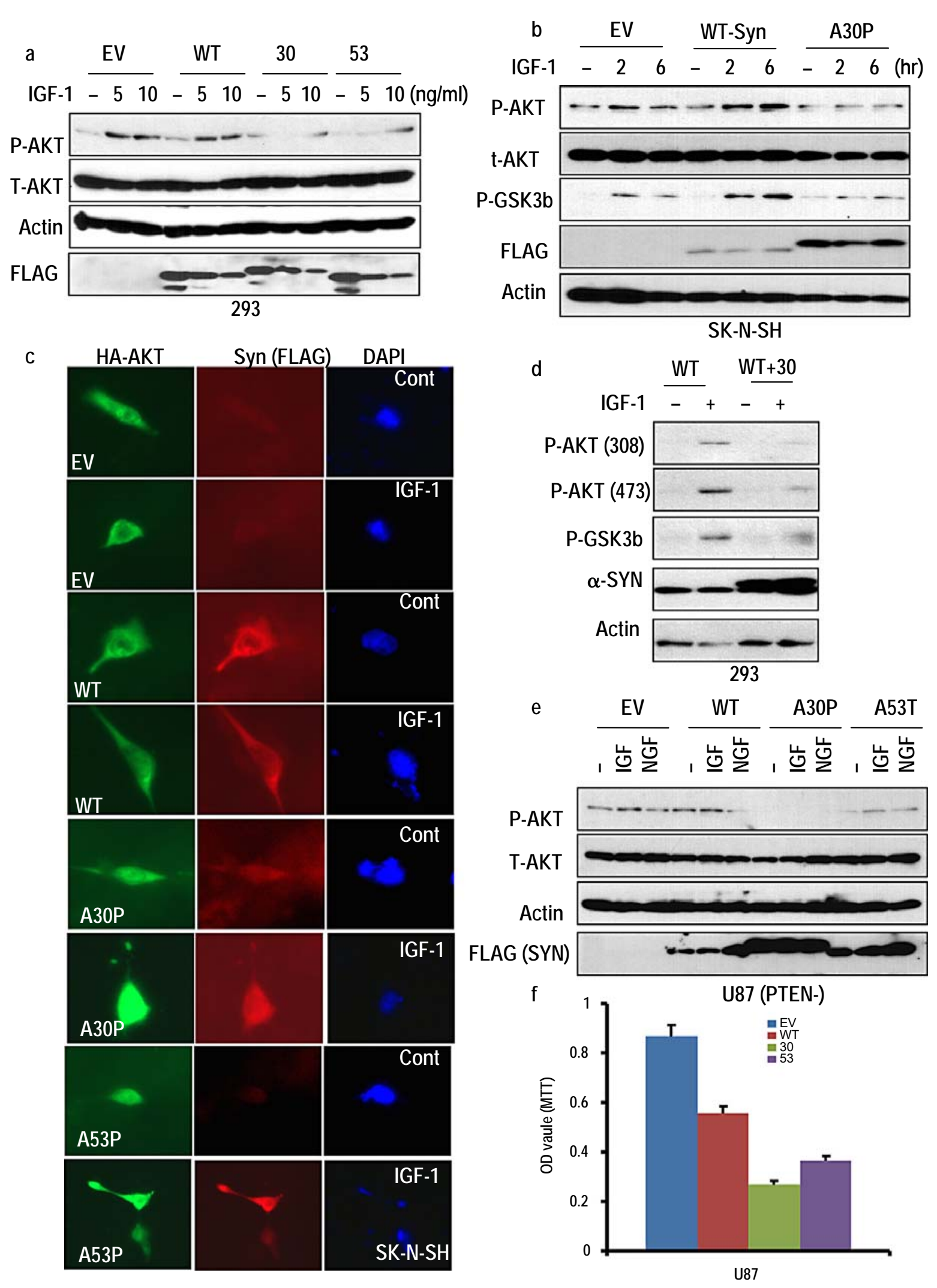
nofluorescence staining. As shown in figure 1c, AKT was concentrated to plasma membrane in response to IGF-1. However, MT-SYN blocked the localization of AKT at plasma membrane. In addition, cytoplasmic AKT formed the speckles or large aggregates in MT-SYN-transfected cells (online suppl. fig. S1, for all online supplementary figures, see www.karger.com/doi/10.1159/000325028). Genetic mutation of SYN is characterized in an autosomal dominant fashion $[1,5]$. Thus, we tested that MTSYN could overwhelm the function of WT-SYN. Activation of AKT by IGF-1 treatment in WT-SYN-transfected cells was blocked by MT-SYN transfection (fig. 1d). Next, we checked the effect of MT-SYN on activated AKT by genetic factor. To address this, we transfected WT- or MT-SYN into the human glioma cell line U87MG, in which AKT is activated by PTEN deficiency [20]. Transfection of MT-SYN could suppress the expression of $\mathrm{p}$ AKT and also blocked the IGF-1 or NGF-1-induced AKT activation (fig. 1e). In addition, MT-SYN obviously reduced the cell viability (fig. 1f). These results strongly suggest that MT-SYNs suppress the activation of AKT and reduce the cell viability. In addition, we could observe the reduction of cell viability in response to WTSYN. We will discuss this later.

Fig. 1. SYN regulates AKT activity. a MT-SYNs block the activation of AKT. 293 cells were transfected with EV, WT, A30P or A53T- $\alpha$-SYN expression vectors for $24 \mathrm{~h}$ and incubated with indicating concentration of IGF-1 for $2 \mathrm{~h}$. Phosphorylation of AKT was detected using p-S473 antibody. Actin was used for loading control. b MT-SYN blocks the AKT-activation in neural cell. SK$\mathrm{N}$-SH cells were incubated with $5 \mathrm{ng} / \mathrm{ml}$ of IGF-1 for indicating time. WT-SYN enhanced the activation of AKT so that phosphorylation of GSK $3 \beta$ was obviously increased. c MT-SYNs block concentration of AKT into plasma membrane in response to IGF-1. Cells were stained with anti-AKT antibodies (green), anti-FLAG (SYN; red), and DAPI (blue) after incubation with IGF-1 $(5 \mathrm{ng} / \mathrm{ml})$ for $30 \mathrm{~min}$. d MT-SYN overwhelms the WT-SYN. SK-N-SH cells were transfected with WT-SYN alone or co-transfected with WTand MT-SYN (A30P) for $24 \mathrm{~h}$. After incubation with IGF-1 (5 ng/ $\mathrm{ml}$ ) for $2 \mathrm{~h}, \mathrm{p}$-AKT (Thr308 and Ser473) was determined. From this experiment, p-AKT indicates p-S473 AKT. e MT-SYN can suppress the activated AKT. U87MG cells (PTEN-deficient cell line) were transfected with WT or MT-SYNs and incubated with IGF-1 or NGF-1 $(5 \mathrm{ng} / \mathrm{ml})$ for $2 \mathrm{~h}$. Although AKT of this cell line is constitutively activated by PTEN deficiency, MT-SYN can suppress the active AKT. $\mathbf{f}$ MT-SYNs suppress the viability of U87MG. Consistently with reduction of p-AKT, cell viability was obviously reduced by A30P and A53T-SYN. Cells were transfected with SYN expression vectors for $24 \mathrm{~h}$ and subjected into MTT assay. We performed the same experiments triple repeated and average values were presented.

$\alpha$-SYN Regulates AKT Activity

\section{$\alpha$-SYN Is Required for AKT Activation}

To further explore the role of SYN in the IGF-1-AKT pathway, we eliminated SYN using si-RNA [17] and measured the activation of AKT in response to IGF-1. Interestingly, si-SYN could suppress the activation of AKT (fig. 2a). In addition, SYN knock-down could block the plasma membrane localization of AKT (fig. 2b). To confirm this, we generated other kinds of si-SYN and performed a similar experiment. In this case, we could also obtain a similar result that elimination of SYN could block the activation and membrane localization of AKT (online suppl. fig. S2). To evaluate the importance of SYN in IGF-1-mediated AKT activation, we compared the effect of TRAF6, which is also known to be essential for IGF-1-induced AKT activation through AKT ubiquitinylation [21]. Consistent with another report [21] and our previous results, elimination of TRFA6 or SYN blocked the IGF-1-induced AKT activation in 293 cells (fig. 2c). To confirm this, we monitored the effect of MTSYN and si-SYN on activation of AKT in normal human fibroblast. Consistent with our previous result, MT-SYN or si-SYN could block the IGF-1-induced AKT activation (fig. 2d). However, in this experiment, we observed the unexpected phenomenon that elimination of SYN can increase the expression of total AKT (fig. 2d). Thus, we checked the location of AKT through immunofluorescence and found that increased AKT was located in the nucleus (fig. 2e). This result implies that SYN is required for proper localization of AKT. In addition, we could observe the reduction of cell viability by elimination of SYN in A549 and U87MG cell lines (fig. 2f). These results strongly suggest that $\alpha-\mathrm{SYN}$ is essential for cell survival.

\section{Overexpression of SYN Also Blocks AKT Activation}

$\mathrm{PD}$ is also evoked by SYN amplification $[5,22]$. This fact implies that excess expression of SYN would block the activation of AKT. To test our hypothesis, we transfected the excessive amount of SYN in SK-N-SH and measured the activation of AKT. In a proper amount of SYN-transfected cells ( $<0.3 \mu \mathrm{g} / \mathrm{ml}$ of WT-SYN), AKT activation was enhanced (fig. 3a), but an excessive SYN (1.2 $\mu \mathrm{g} / \mathrm{ml}$ ) could block the activation of AKT and also reduce the expression of SYN (fig. 3a). In addition, we observed a slight reduction of t-AKT in SYN-transfected cells (fig. 3a). To confirm this, we reperformed a similar experiment with 293 cells. In these cells, overexpression of SYN could block the activation of AKT (fig. 3b). However, SYN was not reduced (fig. 3b). This difference resulted from the different origin of cell lines. Indeed, we have observed a different regulation of $\mathrm{t}$-AKT expression 


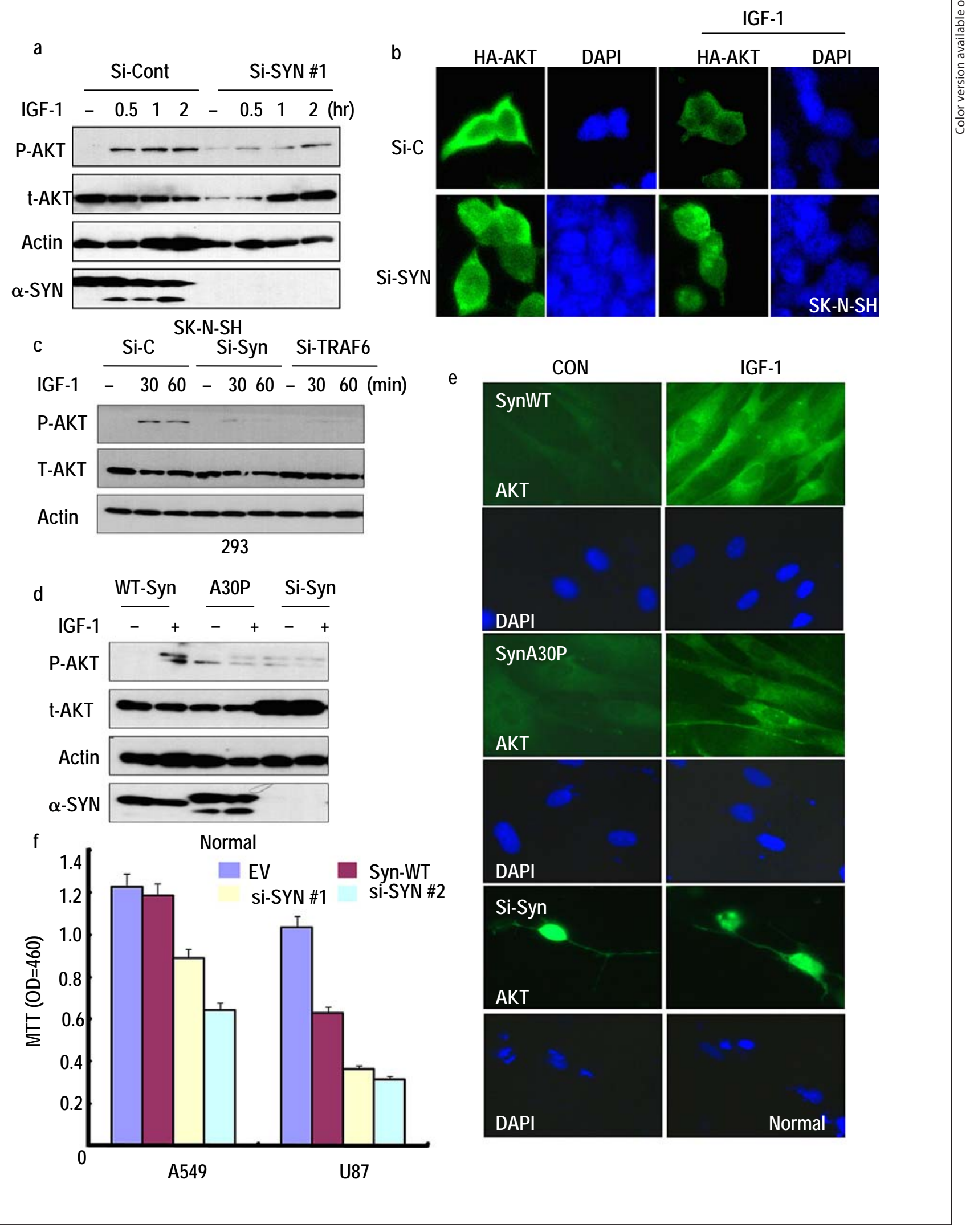


between neuronal cells and non-neural cells by si-SYN (fig. 2c, d). In addition, we previously found that overexpression of WT-SYN could promote cell death (fig. 1f). To find more details, we compared the expression of t-AKT between SK-N-SH and 293 cells after transfection of SYN or si-SYN. Although SYN expression did not show a difference, AKT expression in response to si-SYN showed dramatic changes (fig. 3c). In 293, elimination of SYN promoted the stacking of AKT in RIPA-insoluble fraction (fig. 3c). Instead, MT-SYN promoted the accumulation of AKT in insoluble transfection in SN-K-SH cells (fig. 3c). This result implies that the biological role of SYN is regulation of AKT solubility, which may be required for proper localization. To test this, we transfected AKT and SYN, and monitored the AKT solubility. In EV-transfected cells, AKT was detected in soluble and insoluble fractions in a dose-dependent manner (fig. 3d). WT-SYN suppressed the expression of AKT in an insoluble fraction. In contrast, MT-SYN reduced the solubility of AKT (fig. 3d). Next, we checked the dose effect of SYN on AKT solubility. Interestingly, an excessive amount of SYN also reduced the AKT expression in soluble fraction (fig. 3e). Moreover, MT-SYN did not show a dose-dependent effect (fig. 3e). Next, we measured the expression of p-AKT after transfection of WT or MT-SYN into N2A, SK-N-SH and 293 cell lines to examine the relevance of expression of SYN and p-AKT. In N2A cells, in which endogenous SYN expression is lower than in other cell lines, WT-SYN could induce p-AKT. In contrast, MT-SYN suppressed the basic p-AKT (fig. 3f). Interestingly, SK-N-SH, which showed a modest SYN expression, was not affected by

Fig. 2. SYN is required for AKT activation. a si-RNA against $S Y N$ suppresses the activation of AKT in response to IGF-1. After transfection of si-SYN $(5 \mu \mathrm{g} / \mathrm{ml})$ or si-control for $24 \mathrm{~h}$, SK-N-SH cells were incubated with IGF-1 for indicating time. We could also obtain a similar result for other kinds of si-SYN (online fig. S2). b si-SYN blocks the concentration of AKT into plasma membrane. SK-N-SH cells, transfected with si-RNA against SYN and HA-AKT, were stained with HA (green) and DAPI (blue). c si-SYN blocks the activation of AKT as strong as si-TRAF6. 293 cells were transfected with indicating si-RNA for $24 \mathrm{~h}$ and incubated with IGF-1 for indicating time. $\mathbf{d}$ si-SYN can block the activation of AKT in normal cells. Similarly with MT-SYN, si-SYN blocked the activation of AKT. Moreover, in this cell, si-SYN could increase the expression of total AKT. e si-SYN-induced AKT is located in nucleus. Normal fibroblasts were stained with AKT (green) after transfection with indicating vectors for $24 \mathrm{hr}$. f si-RNAs against SYN suppress cell viability. A549 and U87MG were transfected with two kinds of si-SYN for $24 \mathrm{~h}$. Cell viability was determined by MTT assay.

$\alpha$-SYN Regulates AKT Activity
WT-SYN. In addition, 293 cells showed a reduction of $\mathrm{p}$ AKT in response to WT-SYN transfection (fig. 3f). However, in all kinds of cell lines, MT-SYN suppressed the p-AKT (fig. 3f). These results suggest that a proper amount of WT-SYN enhances AKT function through protecting the aggregation, whereas MT-SYNs or excessively expressed WT-SYN block the AKT activation.

\section{Direct Interaction of AKT and $\alpha-S Y N$}

To obtain more details about the molecular relevance between AKT and SYN, we checked the binding of AKT and SYN through GST pull-down assay. Compared to other recombinant proteins (pVHL or RKIP), AKT was strongly associated with SYN (fig. 4a). We could also observe an interaction of AKT and SYN through IP (fig. 4b). However, MT-SYN did not associate with AKT and AKTKD-SYN interaction was not detected (fig. $4 \mathrm{~b}$ ). To determine the binding domain of AKT, we generated two kinds of AKT proteins (1-126, PH domain; 126-486, kinase domain) [23, 24], and performed the GST pull-down again. From this experiment we could determine that WT-SYN but not MT-SYN was directly interacted with AKT through the kinase domain (fig. 4c). In addition, the $\mathrm{PH}$ domain seemed to weakly contribute to this binding (fig. 4c). This result is consistent with our IP result that AKT-KD did not interact with SYN (fig. 4b). To confirm the direct interaction, we performed the Far-WB analysis [18]. In this experiment, we also obtained a similar result that WT-SYN was strongly bound to AKT, whereas MTSYN did not associate with AKT (online suppl. fig. S3). In addition, AKT-KD-SYN binding seemed to be very weak, so we next checked the effect of MT-SYN on binding between AKT and WT-SYN. To check this, we performed the GST pull-down again after addition of MT-SYN. A30P-SYN could block the interaction between WT-SYN and AKT (fig. 4d). In contrast, addition of WT-SYN could increase the association between MT-SYN and AKT. Moreover, recombinant SYN could form the aggregation, which was increased in a dose-dependent manner (fig. 4d). Considering our above result that MT-SYN did not bind to AKT, the binding between AKT and MT-SYN would be mediated by added WT-SYN. Since AKT is kinase, SYN should be dissociated from activated AKT. To check this, we performed the GST pull-down using recombinant SYN. Interaction between WT-SYN and AKT was reduced in response to IGF-1 (fig. 4e). However, MTSYN-associated AKT was not reduced by IGF-1 (fig. 4e). Indeed, MT-SYN-associated p-AKT was not reduced (fig. 4e). This result strongly suggests that although MTSYN cannot bind to AKT directly, WT-SYN-mediated 


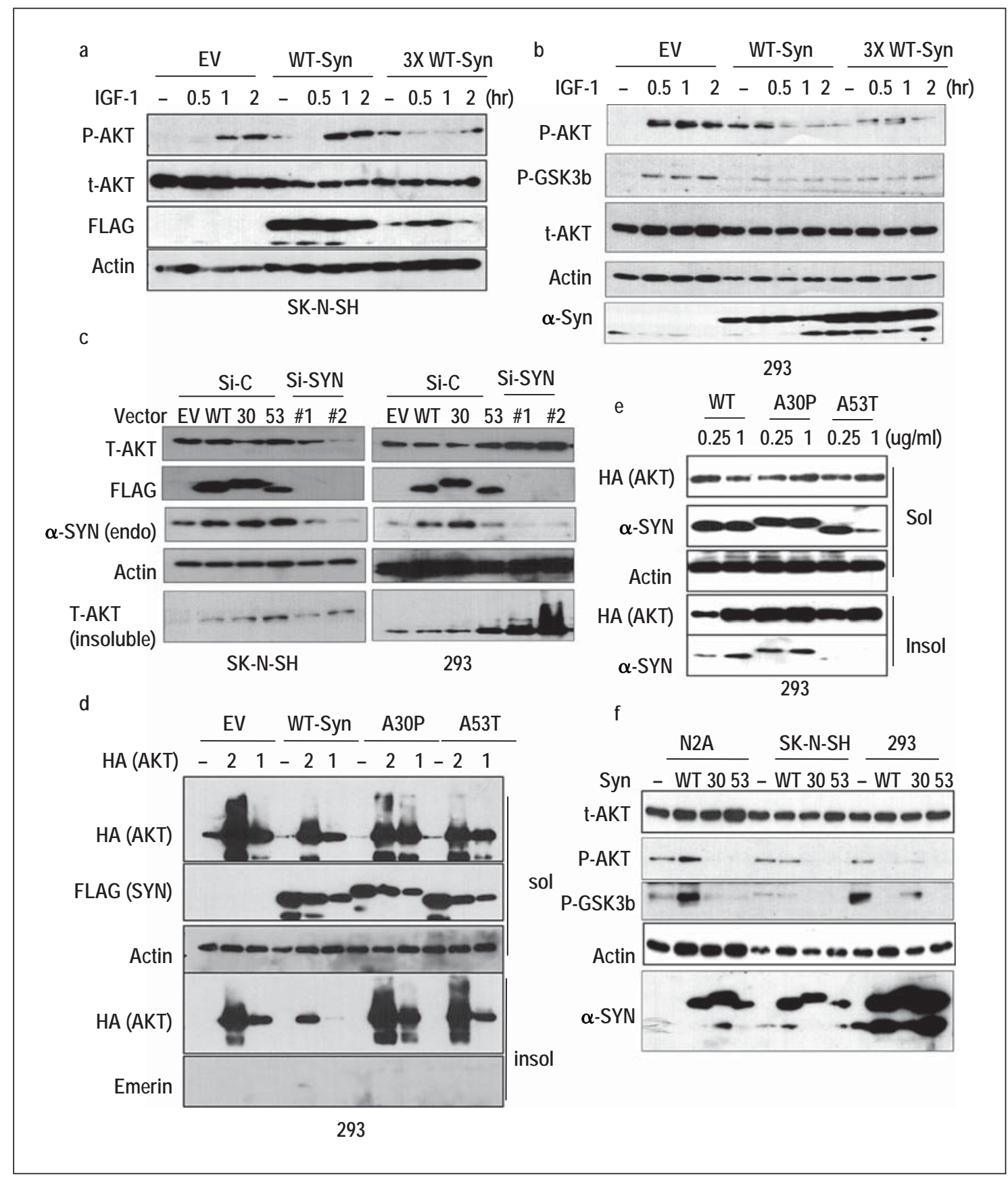

Fig. 3. SYN protects the AKT solubility. a Excessive expression of WT-SYN blocks the activation of AKT. SK-N-SH cells were transfected with 0.3 or $1.2 \mu \mathrm{g} / \mathrm{ml}$ of WT-SYN for $24 \mathrm{~h}$ and incubated with IGF-1 for indicating time. When WT-SYN was excessively expressed, $\alpha$-SYN and p-AKT were obviously reduced. b 293 cells are more sensitive to SYN expression. In 293 cells, $0.3 \mu \mathrm{g} / \mathrm{ml}$ of WT-SYN also suppressed the AKT activation. In addition, $1.2 \mu \mathrm{g} /$ $\mathrm{ml}$ of WT-SYN completely blocked the AKT activation. Moreover, SYN expression was not reduced, differentially from SK-N$\mathrm{SH}$. c Si-SYN increases the expression of total AKT and aggregated AKT in non-neural cell. Cells were transfected with indicating vectors or si-RNA and divided into RIPA soluble and insoluble fractions and were analyzed using WB. Obvious increase of AKT in RIPA insoluble fraction was detected only in 293 cells. d WTSYN enhances the solubility of AKT. In 293 cells indicating concentration of AKT was transfected with WT or MT-SYN for $24 \mathrm{~h}$. Cells were divided into RIPA soluble and insoluble fractions and measured the location of AKT. e Excessive WT-SYN also reduced the AKT solubility. 293 cells were co-transfected with indicating concentration of SYN and HA-AKT and monitored the quantity of soluble or insoluble AKT. f WT-SYN shows the different effect by cell lines. Three kinds of cell lines (N2A, SK-N-SH, and 293) were transfected with indicating SYN expression vectors $(1 \mu \mathrm{g} /$ $\mathrm{ml}$ ) for $24 \mathrm{~h}$ and measured AKT activity by p-Ser473 AKT Ab. 
association with $\mathrm{AKT}$ is not regulated by phosphorylation of AKT. Finally, we measured the cell viability after transfection of si-SYN in SK-N-SH. As mentioned above, si-SYN alone did not show a dramatic suppression effect on cell viability (fig. 4f). However, the IGF-1-mediated protective effect on DNA damage-induced cell death was impaired by si-SYN (fig. 4f) [25]. To introduce the DNA damage, we treated the topoisomerase inhibitor, adriamycin, which is usually treated for double-strand breakage [18]. This result indicates that SYN is required for IGF-1 or the growth factor-mediated protective effect. Considering our results, $\alpha-S Y N$ is essential for AKT activation in response to IGF-1 or growth factors, but if $\alpha$ SYN is mutated or overexpressed, activated or inactivated AKT is sequestered by MT-SYN-AKT complex and IGF1-mediated survival signaling is not propagated (fig. 4g).

\section{Discussion}

Although PD frequently occurs in the elderly population $[1,26]$, a molecular pathological process has not been clearly demonstrated up until now. Since a genetic mutation of SYN has been identified as a causal gene [3, 4], its molecular biological function also remains uncovered. Although mutation frequency of SYN is very rare, SYN mutation-induced familial PD shows general pathological features $[1,27]$. Thus, understanding of molecular function of SYN seems to be important for further investigation of PD. In this study, we found that $\mathrm{SYN}$ is an essential regulator of IGF-1-mediated AKT activation. AKT is important for cell survival and proliferation so that deregulation of AKT signaling will affect cell fate. In fact, MT-SYN can reduce cell viability (fig. 1f). However, MTSYN did not suppress the cell viability in 293 or SK-N-SH in our experimental window (data not shown). This result is consistent with the real phenomenon of $\mathrm{PD}$, that is, the $\mathrm{PD}$ patient is normally developed and pathological symptoms become apparent at least 40 years later affecting dopamineric neuron selectively [28]. Instead, U87MG or A549 cells are grown in an AKT-dependent manner because of PTEN mutation $[19,20,24]$. Thus, we can easily detect the effect of SYN on cell viability (fig. $2 \mathrm{f}$ ). In addition, we have observed the reduction of cell viability by overexpression of WT-SYN in U87MG, a human glioma cell line (fig. 1d, 2f). Considering our result that overexpression of WT-SYN can extraordinarily suppress the pAKT (fig. 3a, b) and an excessive amount of WT-SYN can promote the AKT aggregation (fig. 3d, e), it would be a result from mislocalization or aggregation of AKT which leads to inactivation of AKT. Moreover, reduction of cell viability by MT-SYN was more obvious in U87 than in A549 (fig. 2f). This result would originate from different cellular contexts. U87 has been derived from neuronal glia cells, whereas A549 originates from lung epithelial cells. Thus, they showed the different response to WTSYN (fig. 2f). We can also observe the different response to SYN expression and AKT aggregation between neuronal cells and other cell lines (fig. $3 a-c, f)$. Until now, we did not know the detailed mechanism about the different responses to SYN between neuronal and non-neuronal cells. However, it would contribute to PD because mutation of SYN is a germ-line mutation, whereas neural cells (in particular dopaminergic neuron) are affected [1]. One plausible mechanism is the different kinds of protein elimination pathways between neuronal and non-neuronal cells. Indeed, we recently found that parkin, which is also known to be related with PD, shows a different effect in neural and non-neuronal cells [pers. unpubl. data]. Another possibility is the proliferation ability. Considering the fact that neuron cells cannot proliferate, whereas other kinds of cells can be replaced by proliferation, they should possess a strong and rapid protein degradation mechanism to escape the general toxicity from aggregated protein. In fact, we can observe the different result between 293 and SK-N-SH in AKT reduction in response to SYN overexpression or knock-down (fig. 3a-c). In both cell lines, overexpression of WT-SYN can reduce the tAKT (fig. 3a, b). However, insoluble AKT is increased more obviously in 293 cells (fig. 3c). A more dramatic difference can be obtained from si-SYN (fig. 3c). SYN knockdown induces AKT aggregation apparently in 293 but not in SK-N-SH (fig. 3c).

Considering the fact that MT-SYN or si-SYN can promote AKT aggregation or insolubility (fig. 3d, e), nonproliferating neuron cells should eliminate the aggregated AKT or SYN from the neonatal stage. However, PD occurs in the mid-40s in typical SYN-mutated patients. Thus, in neuronal cells, well-developed protein clearing systems, such as parkin-mediated protein degradation [3, 9], may suppress cell death, but a small amount of protein aggregation, in particular AKT aggregation by SYN mutation stacked during a long period, can lead to neuronal cell death through loss of survival signaling.

We also observed an increase of AKT in the nucleus of normal cells by si-SYN (fig. 2d, e). However, considering the fact that si-SYN increases AKT insolubility (fig. 3ce), it seems to be a non-functional portion. In fact, we examined the FOXO-1 localization, but we did not obtain a meaningful result (data not shown). 


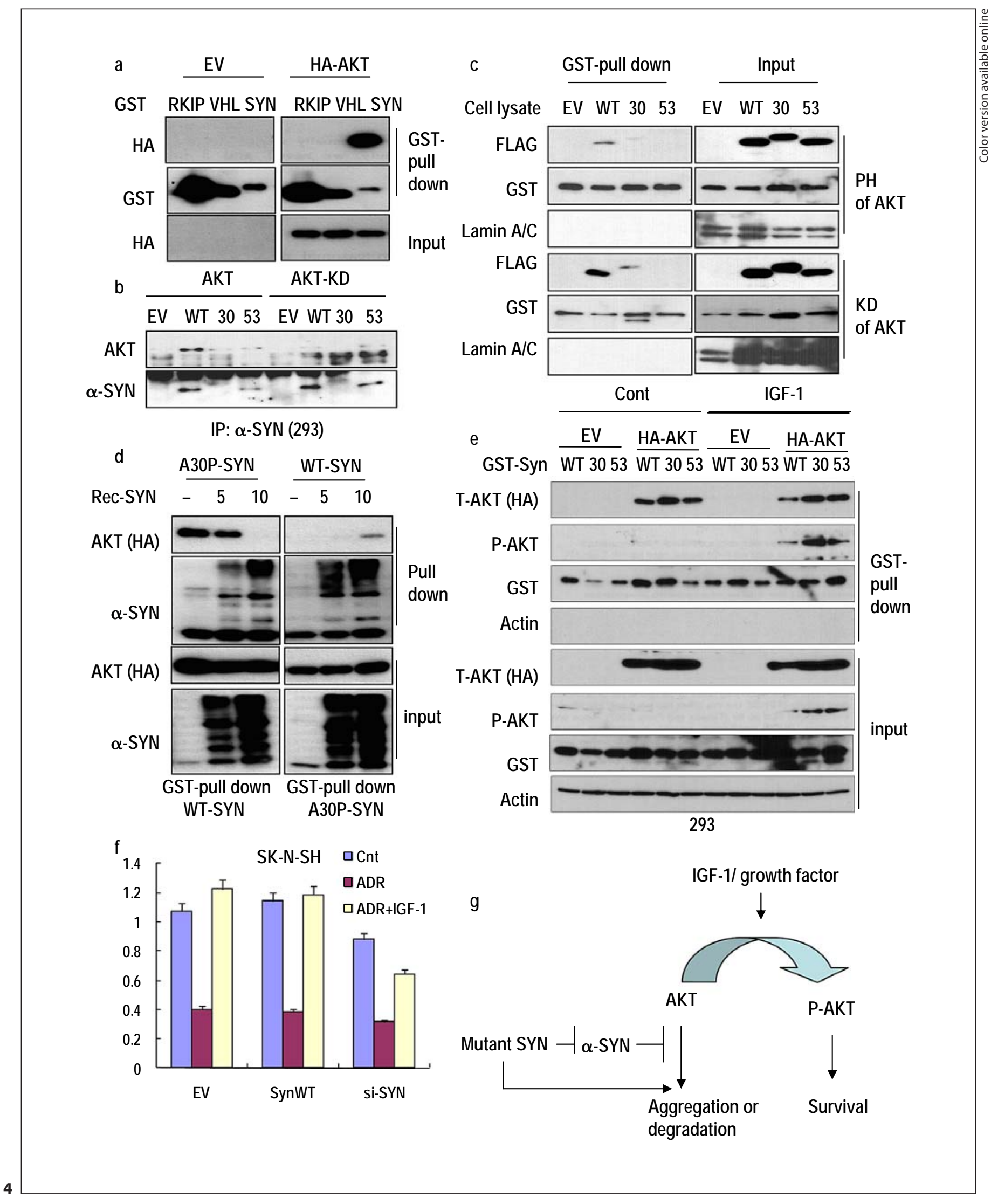


In this study we also show the suppression effect of an excessive amount of WT-SYN on AKT activation (fig. 3a, b). As mentioned above, an extraordinary amount of SYN can promote AKT insolubility (fig. 3d, e) and lead to inactivation of AKT. In fact, we show the different response to AKT activation by the cell context and basal expression of SYN (fig. 3f). We also reveal that SYN directly interacts with AKT (fig. 4), whereas MT-SYN blocks the AKT activity through WT-SYN. This result is also consistent with our well-confirmed genetic theory that MT-SYN is autosomal dominant $[1,5]$. We also revealed that elimination of SYN can increase the sensitivity to DNA damage (fig. 4f). Considering the fact that PD occurred by loss of dopaminergic neuron, the damageprotecting effect of SYN seems to play an important role in neuronal cells.

In addition, we found that excess WT-SYN can reduce the SYN expression itself in a neural cell-specific manner (fig. 3a, b), and we proposed the hypothesis that SYN is

Fig. 4. Direct interaction of AKT and WT-SYN. a SYN interacts with AKT. EV- or AKT-transfected 293 lysates were incubated with GST-RKIP, pVHL or SYN. After GST pull-down, associated AKT was determined by HA antibody. Despite the smallest amount of protein, HA-AKT was detected only in SYN pulldown material. b To confirm the binding between AKT and SYN, we performed the IP. In this time, AKT or AKT-KD-transfected lysate and SYN lysates were separately prepared for preventing the aggregation of AKT. After mixing with these lysates, antibody against SYN was added for IP. From this experiment, we found that WT-SYN was interacted with wild-type AKT. c Kinase domain (KD) of AKT is mainly responsible for binding with WT-SYN. Recombinant PH domain (1-126) and kinase domain (KD, 127-486) of AKT were generated and incubated with SYN-transfected 293 cell lysates for $2 \mathrm{~h}$ at RT. Lamin A/C was used for negative control. d MT-SYN blocks the binding between AKT and WT-SYN. In GST pull-down assay between WT-GST (bead) and AKT (cell lysate), MT-SYN addition suppressed the binding of WT-SYN and AKT. In contrast, addition of WT-SYN could increase the binding between MT-SYN and AKT. We could also observe the increase of high-molecular-weight complexes of SYN following dose of SYN. e WT-SYN-AKT binding is reduced by AKT activation. When cells were activated by IGF1, AKT-WT-SYN binding was reduced. However, binding between AKT and MT-SYN was not reduced by IGF-1 treatment. f Si-SYN suppresses the protective effect of IGF-1 against DNA damage-induced cell death. SN-N-SH cells were transfected with SYN or si-SYN for $24 \mathrm{~h}$ and incubated with $0.2 \mu \mathrm{g} / \mathrm{ml}$ of ADR alone or with IGF-1 (5 $\mu \mathrm{g} / \mathrm{ml})$ for $6 \mathrm{~h}$. Cell viability was determined by MTT assay. $\mathbf{g}$ Simple diagram for summary. WTSYN facilitates AKT activation in response to growth factor, whereas MT-SYN blocks the WT-SYN, leading to impairment of the IGF-1/AKT-signaling axis. required for proper activation of AKT. However, since SYN can be easily aggregated with AKT, SYN expression should be regulated at a low level. If SYN is mutated or amplified by genetic mutation, SYN promotes the AKT aggregation and leads to inactivation of AKT. In nonneuronal cells, the cells can be replaced by proliferation so that a massive cell depletion does not occur. Instead, in the neuron system, aggregated SYN or AKT will be eliminated more effectively. However, long-term accumulated SYN can lead to inactivation of AKT and neuronal cell death. Until now we have not been able to obtain any clues of what kind of factors are critical for determining the fate of SYN.

Taken together, $\alpha-S Y N$ is critical for IGF-1-mediated AKT activation and MT-SYN blocks it. WT-SYN directly binds to the kinase domain of AKT and protects the aggregation of AKT. In contrast, MT-SYN, which is associated with WT-SYN, disrupts the function of WTSYN, resulting in suppression of AKT activation.

\section{Acknowledgements}

This work was supported by Basic Science Research Program through the National Research Foundation of Korea (NRF) funded by the Ministry of Science and Technology [2009-0065237].

References

1 Thomas B, Beal MF: Parkinson's disease. Hum Mol Genet 2007;16:183-194.

-2 Abeliovich A, Schmitz Y, Farinas I, ChoiLundberg D, Ho WH, Castillo PE, Shinsky N, Verdugo JM, Armanini M, Ryan A, Hynes M, Phillips H, Sulzer D, Rosenthal A: Mice lacking $\alpha$-synuclein display functional deficits in the nigrostriatal dopamine system. Neuron 2000;25:239-252.

-3 Licker V, Kövari E, Hochstrasser DF, Burkhard PR: Proteomics in human Parkinson's disease research. J Proteomics 2009;73: $10-29$.

4 Kumari U, Tan EK: LRRK2 in Parkinson's disease: genetic and clinical studies from patients. FEBS J 2009;276:6455-6463.

$\checkmark 5$ Spillantini MG, Schmidt ML, Lee VM, Trojanowski JQ, Jakes R, Goedert M: Alphasynuclein in Lewy bodies. Nature 1997;388: 39-40.

-6 Singleton AB, Farrer M, Johnson J, Singleton A, Hague S, Kachergus J, Hulihan M, Peuralinna T, Dutra A, Nussbaum R, Lincoin S, Crawley A, Hanson M, Maraganore E, Adler C, Cookson MR, Muenter M, Baptista M, Miller D, Blancato J, Hardy J, Gwinn-Hardy K: Alpha-synuclein locus triplication causes Parkinson's disease. Science 2003;302:841. 
7 Yavich L, Jakala P, Tanila H: Abnormal compartmentalization of norepinephrine in mouse dentate gyrus in $\alpha$-synuclein knockout and A30P transgenic mice. J Neurochem 2006;99:724-732.

8 Goedert M: Alpha-synuclein and neurodegenerative diseases. Nat Rev Neurosci 2001; 2:492-501.

-9 Engelender S, Kaminsky Z, Guo X, Sharp AH, Amaravi RK, Kleiderlein JJ, Margolis RL, Troncoso JC, Lanahan AA, Worley PF, Dawson VL, Dawson TM, Ross CA: Synphilin-1 associates with $\alpha$-synuclein and promotes the formation of cytosolic inclusions. Nat Genet 1999;22:110-114.

10 Wakabayashi K, Engelender S, Yoshimoto M, Tsuji S, Ross CA, Takahashi H: Synphilin1 is present in Lewy bodies in Parkinson's disease. Ann Neurol 2000;47:521-523.

-11 Offen D, Shtaif B, Hadad D, Weizman A, Melamed E, Gil-Ad I: Protective effect of insulin-like-growth-factor-1 against dopamine-induced neurotoxicity in human and rodent neuronal cultures: possible implications for Parkinson's disease. Neurosci Lett 2001;316:129-132.

12 Kao SY: Rescue of $\alpha$-synuclein cytotoxicity by insulin-like growth factors. Biochem Biophys Res Commun 2009;385:434-438.

13 Valente EM, Abou-Sleiman PM, Caputo V, Muqit MM, Harvey K, Gispert S, Ali Z, DelTurco D, Bentivoglio AR, Healy DG, Albanese A, Nussbaum R, González-Maldonado R, Deller T, Salvi S, Cortelli P, Gilks WP, Latchman DS, Harvey RJ, Dallapiccola B, Auburger G, Wood NW: Hereditary earlyonset Parkinson's disease caused by mutations in PINK1. Science 2004;304:11581160.
14 Xu J, Zhong N, Wang H, Elias JE, Kim CY, Woldman I, Pifl C, Gygi SP, Geula C, Yankner BA: The Parkinson's disease-associated DJ-1 protein is a transcriptional coactivator that protects against neuronal apoptosis. Hum Mol Genet 2005;14:12311241.

15 Bonifati V, Rizzu P, van Baren MJ, Schaap O, Breedveld GJ, Krieger E, Dekker MC, Squitieri F, Ibanez P, Joosse M, van Dongen JW, Vanacore N, van Swieten JC, Brice A, Meco G, van Duijin CM, Oostra BA, Heutink P: Mutations in the $D J-1$ gene associated with autosomal recessive early-onset parkinsonism. Science 2003;299:256-259.

16 Martins LM, Morrison A, Klupsch K, Fedele V, Moisoi N, Teismann P, Abuin A, Grau E, Geppert M, Livi GP, Creasy CL, Martin A, Hargreaves I, Heales SJ, Okada H, Brandner S, Schulz JB, Mak T, Downward J: Neuroprotective role of the Reaper-related serine protease $\mathrm{HtrA} / \mathrm{Omi}$ revealed by targeted deletion in mice. Mol Cell Biol 2004;24:98489862.

17 Fountaine TM, Wade-Martins R: RNA interference-mediated knockdown of $\alpha$-synuclein protects human dopaminergic neuroblastoma cells from $\mathrm{MPP}^{+}$toxicity and reduces dopamine transport. J Neurosci Res 2007;85:351-363.

18 Lee SH, Lee SJ, Jung YS, Xu Y, Kang HS, Ha NC, Park BJ: Blocking of p53-Snail binding, promoted by oncogenic K-Ras, recovers p53 expression and function. Neoplasia 2009;11: 22-31.

19 Downward J: Mechanisms and consequences of activation of protein kinase B/Akt. Curr Opin Cell Biol 1998;10:262-267.

20 Cheney IW, Johnson DE, Vaillancourt MT, Avanzini J, Morimoto A, Demers GW, Wills KN, Shabram PW, Bolen JB, Tavtigian SV, Bookstein R: Suppression of tumorigenicity of glioblastoma cells by adenovirus-mediated MMAC1/PTEN gene transfer. Cancer Res 1998;58:2331-2334.
1 Yang WL, Wang J, Chan CH, Lee SW, Campos AD, Lamothe B, Hur L, Grabiner BC, Lin X, Darnay BG, Lin HK: The E3 ligase TRAF6 regulates Akt ubiquitination and activation. Science 2009;325:1134-1138.

22 Miller DW, Hague SM, Clarimon J, Baptista M, Gwinn-Hardy K, Cookson MR, Singleton AB: Alpha-synuclein in blood and brain from familial Parkinson disease with SNCA locus triplication. Neurology 2004;62:18351838.

23 Kumar CC, Madison V: AKT crystal structure and AKT-specific inhibitors. Oncogene 2005;24:7493-7501.

24 Brugge J, Hung MC, Mills GB: A new mutational AKT activation in the PI3K pathway. Cancer Cell 2007;12:104-107.

25 Zaka M, Rafi MA, Rao HZ, Luzi P, Wenger DA: Insulin-like growth factor-1 provides protection against psychosine-induced apoptosis in cultured mouse oligodendrocyte progenitor cells using primarily the PI3K/Akt pathway. Mol Cell Neurosci 2005; 30:398-407.

26 Dorsey ER, Constantinescu R, Thompson JP, Biglan KM, Holloway RG, Kieburtz K, Marshall FJ, Ravina BM, Schifitto G, Siderowf A, Tanner CM: Projected number of people with Parkinson disease in the most populous nations, 2005 through 2030. Neurology 2007;68:384-386.

27 Polymeropoulos MH, Lavedan C, Leroy E, Ide SE, Dehejia A, Dutra A, Pike B, Root H, Rubenstein J, Boyer R, Stenroos ES, Chandrasekharappa S, Athanassiadou A, Papapetropoulos T, Johnson WG, Lazzarini AM, Duvoisin RC, Di Iorio G, Golbe LI, Nussbaum RL: Mutation in the $\alpha$-synuclein gene identified in families with Parkinson's disease. Science 1997;276:2045-2047.

28 Hardy J, Cookson MR, Singleton A: Genes and parkinsonism. Lancet Neurol 2003;2: 221-228. 